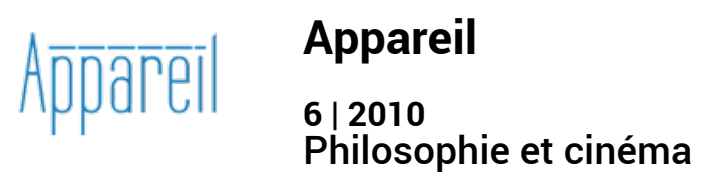

\title{
Geste cinématographique et cinéma documentaire
}

\section{Philippe Roy}

\section{(2) OpenEdition}

Journals

Édition électronique

URL : http://journals.openedition.org/appareil/1087

DOI : 10.4000/appareil. 1087

ISSN : 2101-0714

Éditeur

MSH Paris Nord

\section{Référence électronique}

Philippe Roy, «Geste cinématographique et cinéma documentaire », Appareil [En ligne], 6 | 2010, mis en ligne le 07 octobre 2010, consulté le 10 décembre 2020. URL : http://journals.openedition.org/ appareil/1087 ; DOI : https://doi.org/10.4000/appareil.1087

Ce document a été généré automatiquement le 10 décembre 2020.

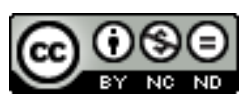

Appareil est mis à disposition selon les termes de la Licence Creative Commons Attribution - Pas d'Utilisation Commerciale - Pas de Modification 4.0 International. 


\title{
Geste cinématographique et cinéma documentaire
}

\author{
Philippe Roy
}

1 J'établirai ici un concept de geste cinématographique que je spécifierai ensuite comme documentaire. Il sera essentiellement question de la modalité d'expression cinématographique et non de ce qui se présente avec le geste singulier de tel ou tel artiste-cinéaste. Je le ferai seulement un peu dans le cadre du cinéma documentaire. Ce sont quelques traits d'un mode d'approche gestuel du cinéma documentaire qu'il m'importe donc de dégager dans cet article. Avec ceci qu'en plus ces traits permettent de croiser désir et politique dans le champ même du cinéma et de sa technicité.

\section{Le geste cinématographique}

\section{1. Le geste de traçage}

2 Faisons preuve de naïveté. Nous regardons un film, des ondes lumineuses et sonores nous traversent, nous sommes en elles mais elles ne nous heurtent pas, leurs masses sont nulles. La composition des ondes lumineuses dépend du cadrage (angle compris) et de son contenu, du réglage des couleurs aussi, telle est la vue corrélative d'un point de vue, celui du filmeur ${ }^{1}$. Ce point de vue peut être fixe ou mobile (mouvements d'appareil dont le zoom) et même absent dans les situations graphiques (monochromes, certains dessins, etc.) ou seulement sonores. Le point de vue est ce par rapport à quoi seront référés d'autres points de vue incarnés à l'image (par exemple point de vue d'où tel acteur regarde) ou d'autres vues possibles ou sons effectifs en dehors du cadre (hors champ). Le point de vue ordonne donc une multiplicité d'autres points de vue implicites. Mais, par projection de notre corps propre, au point de vue du filmeur est souvent couplée une deuxième référence où le filmeur est situé qui est le référentiel terrestre ${ }^{2}$, le hors-champ le suppose.

3 J'appelle «traçage" d'un film le passage par les doublets points de vue/vues accompagnés ou pas par des sons, selon cette première acception le traçage est dit tel 
parce qu'il connecte des points de vue, plus profondément il est tel car il dirige la progression dans le temps. Si bien que cette progression peut très bien avoir lieu sans point de vue lorsque le traçage n'est que sonore ou/et pictural (le filmeur n'est plus que sonorisateur ou graphiste). Un plan est un traçage filmique continu formant un tracé, il lui appartient donc d'avoir une durée. Le plan dépend d'une ligne de connexion des points de vue, c'est une ligne de vues (lorsque le point de vue est fixe la ligne est réduite au point) ou d'une ligne sonore ou/et picturale. Le traçage est le geste du filmeur pour chaque plan.

4 Je n'ai mis l'accent que sur une moitié de ma définition du traçage, ce geste n'est en effet pas seulement celui du corps agissant du filmeur mais bien aussi celui de son œil et de son oreille (comme je l'ai dit pour la ligne sonore). Écartons tout de suite plusieurs malentendus possibles, je ne comprends pas par "geste » les mouvements de caméra du filmeur, comptent aussi bien le choix du contenu et de son cadrage, le réglage des couleurs, le découpage et le réglage de la bande son et même le choix du matériel technique ${ }^{3}$. Bien plus le geste n'est pas cet ensemble technique. Les éléments de cet ensemble sont des opérations qui ont réalisé le geste alors que ce dernier est ce qui à la fois motive ces réalisations et s'enveloppe avec chaque plan. Mais surtout entendons bien qu'au geste appartiennent les intensités par lesquelles il s'exerce, les intensités lumineuses, sonores et leurs intensités impliquées: telles celles des mouvements correspondant aux variations du contenu et aux changements de point de vue, celles de profondeur, celles associées au contenu (poids, forces, chaleur par exemple) ou associées aux visages, aux voix (intensités d'affects). Le geste de traçage a donc lieu tout le long d'un plan, même quand celui-ci est fixe, puisqu'il compose les changements intérieurs à la vue et aux sons et le changement de points de vue, les premiers pouvant avoir lieu sans le deuxième ce pourquoi le geste de traçage peut coïncider avec un geste filmé (par exemple chaque plan du geste « Hulot » chez Jacques Tati).

5 Les rapports variables de ces intensités deviennent répartition extensive variable

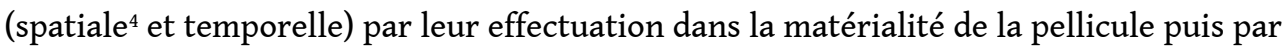
la projection de la suite des photogrammes sur l'écran (condition de notre synthèse temporelle) et enfin par leur constitution en nous comme tracé. Le plan est donc à la fois le tracé résultant du traçage et la trace mobile du contenu filmé, c'est-à-dire d'un présent lors du filmage (présent du direct) ou d'un ancien présent. Ou pour le dire autrement le plan est pensé comme trace mobile en tant que réception d'un contenu en lequel s'exerce le traçage, c'est si l'on veut la face «photogrammes » du film et pensé comme tracé en tant qu'émission du traçage, c'est la face « écran » du film. Mais trace et tracé sont l'un dans l'autre, expression et contenu sont indiscernables, pilotés par le

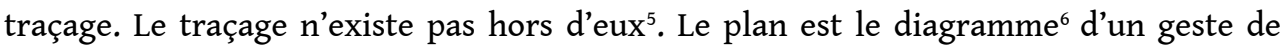
traçage en train de se faire. Un plan nous place dans son diagramme et sous son geste.

\section{2. Le geste de coupure}

6 Je n'ai évidemment pas terminé ma présentation du geste cinématographique car les tracés doivent être raccordés et même composés dans le cas de tracés superposés (images/images ou images/sons) ou se partageant l'écran. Telle est la fonction du montage. Mais le terme de raccord est trompeur car il ne signifie pas ici le rétablissement d'une jonction. Au contraire chaque raccord réalise toujours une coupure. Nous retrouvons ici la distinction entre le geste et l'opération. Raccorder deux plans est l'opération qui réalise le geste de coupure, le raccord est la trace de la coupure. 
La coupure est l'entre-deux, le vide actif qui sépare et réunit deux plans, deux tracés. En même temps qu'elle fait passer un plan elle en fait advenir un nouveau, cette coupure répétée ordonne les plans ${ }^{7}$.

Bien plus, le geste de coupure est une auto-affection du film car ces plans d'un nouveau présent et de l'ancien présent ne sont pas sans retentir l'un sur l'autre et même, d'une façon plus ou moins intense pour chacun, ce sont tous les anciens plans qui retentissent sur le nouveau plan et lui sur eux, ce retentissement se mesurant aux attentes qu'induisent les anciens plans confrontées à la nouveauté d'un autre, les attentes étant à chaque fois remaniées après chaque retentissement. Lorsqu'une attente est comblée le retentissement est peu intense c'est même la raison pour laquelle le geste de coupure se fait bien souvent oublier sous le raccord ${ }^{8}$. Enfin la suite des gestes de coupure détermine la temporalité du film (son rythme) incluant un certain traitement des temporalités des contenus filmés, de leurs histoires, d'où découleront des formes de narration.

Où a lieu ce retentissement ? Ce ne peut être dans le champ du traçage puisque le geste de coupure réunit et sépare des tracés, il n'est pas dans le flux du passage mais sur le lieu du passage. En effet pas de passage sans un lieu de passage immobile qui ne passe pas, pas de passage de la douane sans une douane comme lieu fixe du passage. Le lieu du passage est ici notre mémoire car elle ne serait pas telle si elle passait avec ce qu'elle mémorise. Avec le geste de coupure la mémoire est mise à l'épreuve, prise entre attentes et nouveauté. C'est par là que le geste de coupure peut donner à penser. Notre mémoire est le lieu des retentissements fendue par les gestes de coupure. Le cinéma ne va pas vers le spectateur sans venir de lui.

\section{3. Niveau des gestes}

9 Le geste cinématographique est donc une composition de gestes de traçage et de coupure ${ }^{9}$. Or la substance d'expression cinématographique est telle que le tracé vient avec son traçage et le raccord avec sa coupure. Le geste cinématographique affleure dans la substance en laquelle et par laquelle il s'enveloppe et s'exerce, il affleure dans ses ondes lumineuses et sonores. La masse nulle de cette substance la distingue du niveau matériel massique où les gestes s'effectuent, se réalisent et deviennent des actes. Le geste cinématographique se détache des actes et des opérations ${ }^{10}$ de la réalisation du film et leur survit. Alors que le geste du peintre s'est réalisé, celui de la danse ou du théâtre se réalise, le geste du cinéma s'effectue de façon minimale, comme retenu dans sa matérialité sans épaisseur, fantomatique, épidermique, dans un suspens qui l'empêche de retomber dans le monde des corps. Contrairement à la danse, la peinture ou le théâtre, la réalisation du film précède l'expression du geste cinématographique ${ }^{11}$ (alors que la réalisation du tableau succède au geste qu'il exprime et que les gestes de la danse ou du théâtre sont en simultanéité avec leurs réalisations). Le geste cinématographique peut se maintenir au niveau du geste, réalisation arrêtée ${ }^{12}$. Il révèle le niveau des gestes en tant que gestes, niveau vers lequel les arts de réalisation remontent aussi, à leur façon, puisqu'ils nous invitent à extraire de leurs actes leur part gestuelle ${ }^{13}$, part spirituelle et affective. Par leur levée gestuelle les arts de réalisation pervertissent la réalisation ce pourquoi leurs actes décrochent du plan commun des actes, seuls les gestes de leurs actes frappent les spectateurs. Le geste 
cinématographique est lui d'emblée en levée, il virtualise l'acte ${ }^{14}$ (alors que dans l'autre sens un acte réalise un geste).

Si on convient de dire qu'une perception engage des rapports entre des corps massiques (ceux du percevant et du perçu) insérée dans le champ des actions, alors le cinéma relève moins de la perception, nous ne le rapportons peu à ce corps qu'est l'écran et encore moins aux photogrammes, il est surtout imaginaction ${ }^{15}$, imaginomoteur. Sans cela il ne pourrait pas venir brouiller la perception avec par exemple des spectres et des effets spéciaux ou nous présenter des images se présentant comme celles d'un passé. De même que la perception est au milieu des choses massives, l'imagination est au milieu des ondes de masses nulles. Perception et imagination ne sont pas les facultés d'un sujet, ce sont les corrélats subjectifs d'un accès à un certain niveau de la réalité ${ }^{16}$, c'est donc moins d'imagination qu'il faudrait parler mais d'images optiques et sonores actives-à-activer, avec Gilbert Simondon il faut concevoir « l'image comme réalité intermédiaire entre objet et sujet, concret et abstrait, passé et avenir ${ }^{17}$ ». Notre orientation philosophique est dans la filiation de la «Naturphilosophie»: la nature est en nous comme nous sommes en elle ${ }^{18}$, nous ne sommes pas devant elle (refus de s'en tenir au dualisme sujet/objet). Le cinéma est comme le rêve, il nous fait vivre les intensités et les enveloppements de ses gestes en les séparant de leur réalisation par notre corps, les effectuations ne sont plus seulement que naissantes en nous, tout en pénétrant dans le champ de nos mémoires avec des intensités variables de retentissement, auto-affections filmiques ${ }^{19}$.

\section{Premières spécificités de gestes documentaires}

\section{1. Mise en scène ?}

11 Le geste cinématographique tel que je l'ai décrit jusque là concerne autant le documentaire que la fiction. Pensons à présent leurs lignes de séparation quitte à modérer la séparation quand cela sera nécessaire et même à penser plus tard leur ligne d'indiscernabilité. La première différence qui vient à l'esprit est évidemment en lien avec le contenu des films. Dans le cinéma de fiction ce qui est filmé existe pour le film, sa durée de vie est égale à la durée du tournage, dans le cinéma documentaire ce qui est filmé a déjà une vie avant que n'intervienne le filmeur, qui continuera après. Si bien que dans la fiction le geste cinématographique est indissociable d'une mise en scène du contenu alors que le documentaire se confronte à un contenu brut, imprévisible, le champ des intensités est donc plus préparé dans la fiction (on choisira tels éclairages, telle couleur pour le costume, etc.).

Mais modérons de suite l'arbitrage du partage par la présence de la mise en scène, car il est des réalisateurs tel Robert Bresson qui contrarie tout jeu de mise en scène par l'apport d'acteurs non comédiens (qu'il appelle des modèles) qui ne jouent pas ${ }^{20}$, « ton imagination visera moins les événements que les sentiments, tout en voulant ces derniers aussi documentaires que possible ${ }^{21}$ » et inversement il est des réalisateurs de documentaires tel Johan Van der Keuken qui propose parfois une mise en scène aux gens qu'il filme ou tel Claudio Pazienza qui se met en scène avec ses parents ${ }^{22}$. De plus puisque le contenu peut être préparé, ne concluons pas trop vite à une plus grande adéquation entre le contenu et l'expression cinématographique dans le cinéma de fiction que dans le cinéma documentaire. Comme si le filmeur documentaire se 
contentait d'enregistrer le réel qui se présente là devant lui or il n'y a pas LE réel indépendant de tout point de vue. Le contenu est toujours celui d'un film, il est donné par le geste. Comme le dit Serge Daney un film implique toujours une certaine manière de montrer.

\section{2. Jauge du geste documentaire}

Revenons en amont de l'argument de la mise en scène, le cinéma documentaire se fait sur fond d'une vie qui lui pré-existe. Or le statut de cette vie est identique aux nôtres, spectateurs. Nous pré-existons à tout film et nous sommes aussi, comme les personnages filmés, des sujets dont les paroles, les actes sont indissociables des vies de nos corps. Comme l'écrit Jean-louis Comolli :

J'y verrai volontiers la principale ligne de partage entre cinéma de fiction et cinéma documentaire : dans un cas et dans l'autre, il ne s'agit pas de la même relation entre corps et personnage, inscription et narration. À l'adéquation documentaire du corps, de la parole, du sujet, du personnage répondent la dissociation et la recomposition fictionnelles de ces mêmes éléments, disjoints, divergents, que le travail du comédien (avec celui de la mise en scène) tente de rendre plus solidaires, plus articulés, plus cohérents ${ }^{23}$.

14 À cause de cette identification de statut entre les personnages et les spectateurs, les intensités affectives du geste cinématographique seront évaluées, jugées, il y a un compatir. Dans un documentaire de Sylvain George ${ }^{24}$ nous est donné à voir le brouillage des empreintes digitales par des immigrants clandestins, ils râpent leurs bouts de doigts avec une vis chauffée. La scène dure longtemps, très longtemps, nous endurons ce que le geste cinématographique enveloppe (auquel appartient la durée du plan), pas question de se dire « ce n'est que du cinéma! ». En donnant à voir, à entendre, le geste donne à évaluer. Mais en tant qu'il donne à évaluer de telle ou telle manière c'est le geste lui-même qui sera considéré comme plus ou moins bien jaugé par tel ou tel spectateur. Le geste du plan du film de Sylvain Georges était-il bien jaugé ? Sa longue durée n'a-t-elle pas dépassé le cap de l'insistance de trop?

\section{3. Geste documentaire d'intervention}

Dans le cinéma documentaire le filmeur intervient dans son film. Ne serait-ce qu'à un premier niveau rudimentaire, la simple manière d'être du filmeur produit des effets sur le filmé ou inversement le filmé fait réagir le filmeur si bien qu'indirectement sa réaction est impliquée par son geste cinématographique. Cela est parfois très manifeste, par exemple dans la première séquence de Et la vie, Denis Gheerbrant, caméra en main, suscite une demande chez un habitant de la rue qu'il sillonne, celui-ci va l'inviter à venir filmer dans sa maison. Le corps, la parole du filmeur sont même souvent des composantes du film. Comme la main d'Agnès Varda dans Les glaneurs et la glaneuse mais aussi celle de Denis Gheerbrant placée devant l'objectif de sa caméra à la demande d'une femme qui a honte du lieu insalubre où elle loge $e^{25}$, sa main met en œuvre une réaction à l'effet que produit chez elle son arrivée. Pensons aussi au visage et la voix d'Avi Mograbi.

Le filmeur peut même devenir implicitement le principal filmé du film. Dans un film au titre on ne peut plus explicite Le filmeur, Alain Cavalier filme le monde qui se rapporte à lui en montrant comment ce monde se rapporte à lui. Par exemple filmant sa mère qui 
dort, il la filme surprise par son intrusion invisible (donc comment elle se rapporte à son intrusion), même chose avec sa femme qui dort. Dans ce film le traçage fait signe vers son traceur et les raccords sont parfois autant les traces de ses gestes de coupure que celles des coupures de sa vie (des dix ans où ont été prélevées les traces). Ce lien circulaire entre filmeur et filmé que je viens sommairement d'exemplifier me porte à dire que le geste de traçage documentaire est un geste d'intervention. Bien plus, ce lien circulaire est celui de la rencontre entre filmeur et filmé, il suppose l'entre-deux, où s'insère véritablement l'intervention. Filmeur et filmé sont engagés dans un devenir inconnu d'eux. «La non-maitrise du documentaire apparaît comme la condition de l'invention. C'est la puissance réelle de ce monde qui éclate à travers elle ${ }^{26} »$. Le geste de traçage saisit chaque événement qu'il suscite, il s'enveloppe et se développe dans et par son tracé. Les gestes de coupure viennent rythmer les développements en faisant retentir les événements, dans leurs entre-deux. La suite des coupures donne du large à toutes les interventions, elle fait coexister leurs espaces-temps, tout en étant autoaffection du film comme du filmeur, retentissant en nous. Par exemple le geste de Boris Lehman dans Babel. Lettre à mes amis restés en Belgique ${ }^{27}$, plaçant une coupure entre les événements qui précèdent son voyage au Mexique et les événements de son retour, fait coexister ces espaces-temps et retentir l'un sur l'autre les tracés de l'avant et de l'après, le retentissement donnant à penser : que s'est-il passé entre ? Est-il vraiment allé au Mexique? Quels affects produit en nous cette coupure?

\section{4. La résonance de la singularité au lieu du rapportage}

On opposera reportage et cinéma documentaire si on convient d'appeler "geste cinématographique de reportage ", le geste calibré, répondant à des normes de prescripteurs, selon des formats télévisuels par exemple et même des jauges affectives déterminées qui viennent confirmer des évaluations sociales pré-données (humanitaires ou sécuritaires par exemple). Si bien que cela implique que le filmeur s'efface derrière ces normes et que de plus il n'engage en rien un geste d'intervention. La fausse prétention à l'objectivité du reportage n'est telle que par l'effacement de la subjectivité du filmeur. Mais cette objectivité est de toute façon celle d'un geste, fut-il calibré et non intervenant ou du moins pour reprendre une distinction du Moyen Âge, c'est une geste (gesta) et non pas un gestus. En effet « dans la tradition antique qui peu à peu s'impose durant le Haut Moyen Âge, la notion de gestus suppose la possibilité d'une maîtrise et d'une responsabilité individuelle des gestes. Dans les gesta, dans la geste, au contraire, l'individu dépend entièrement du groupe, monastère ou lignage. Pas plus que le sens de l'histoire, celui de ses actions ne lui appartient vraiment ${ }^{28}$. » Si bien qu'il implique que nous visions le contenu filmique selon cette geste sociale, selon sa monstration. L'expression standardisée tend à devenir indifférente à son contenu, il y a comme une vitre entre l'expression et le contenu, ils ne sont plus indiscernables, le contenu est seulement rapporté à une forme d'expression. Tout reportage est donc un rapportage. Par sa répétition la geste forge la conduite cinématographique du spectateur si bien qu'à contrario on peut qualifier le gestus documentaire de contreconduite.

18 À la différence d'un reportage, un film documentaire a la capacité de faire résonner un monde. Et ceci est favorisé lorsqu'une personne filmée parle, les paroles de celle-ci faisant naître des images qui ne sont pas à l'écran. La parole filmée est alors le vecteur d'un autre film en nous. La parole et la visagéité du corps parlant font résonner la 
source de leur épanchement : un monde. Il y a tout un art du voisinage dans le cinéma, on ne filme jamais aussi bien un monde ou quelqu'un que lorsqu'il est l'horizon-limite de la suite des filmages de ses entours. Dans sa grande fresque sur Marseille, Denis Gheerbrant fait revivre des mondes en recourant aux entours de personnes filmées. Ces filmés ne sont pas choisis au hasard, ils sont ceux qui sont capables de faire résonner ce monde d'où ils parlent et qu'ils font encore parler, ceux dont le grain de la voix est aussi la graine d'un monde. Les filmés sont des singularités qui comme telles font résonner leurs voisinages, leurs mondes. Et ces singularités résonnent en nous, nous renvoyant au monde qu'elles affirment et à sa perte, à la nécessité donc pour nous de ne plus perdre et donc de faire monde, de voisiner singulièrement avec d'autres. Quand il en est ainsi le cinéma documentaire est moins un rappel qu'un appel aux actes.

\section{Gestes cinématographiques politiques}

\section{1. Geste de résurrection d'un réel}

19 Cela m'amène à parler de l'implication politique du geste cinématographique. Puisque le geste affleure dans sa substance ondulatoire, il ne va pas être sans induire des gestes dans notre corps imaginacteur. Et ces gestes peuvent être politiques. Le geste de coupure peut ainsi permettre le retentissement de tracés séparés spatialement même et surtout quand il n'y a plus de trace dans la réalité, de trace réalisée. Le geste du film Katyn d'Andrzej Wajda fait retentir les segments dissociés du massacre de masse par lesquels transitent les victimes polonaises, chaque segment étant une étape de ce massacre à la chaîne, aboutissant à un charnier enterré qui doit éliminer toute trace. Son geste nous montre cette réalité telle qu'elle n'a pu être vue par personne, pas même par un officier russe. Ce geste remédie à la disparition. Le dernier segment lorsque y arrivent directement certaines victimes est tel que celles-ci aperçoivent leurs compatriotes morts dans le charnier, en même temps qu'ils signent leur appartenance polonaise (par une prière par exemple), le geste d'Andrzej Wajda faisant ainsi retentir avec ce plan tous les plans précédents et conditionne notre attente de ceux qui viendront. Par là, et avec Wajda, c'est tout le peuple polonais que nous faisons vivre, nous spectateurs actuels, en notre mémoire, pour toujours. La fiction fait documentaire, faisant même coïncider en sa fin un fragment documentaire qu'est le cahier de notes ayant appartenu à une victime et la fin de l'écriture de ses impressions journalières que le film présentait avant sa mort. La fiction cinématographique ressuscite un réel et son geste de résurrection réactive un geste politique patriotique. De plus le geste cinématographique en prélevant le traçage de l'écriture en isole le geste-se-faisant, qui devient ici le suivi d'une mémoire polonaise s'auto-affectant. De même que le geste cinématographique se détache de la réalisation, il détache pour cette raison même les autres gestes qu'il filme, de leur réalisation. Le geste cinématographique toujours en train de se faire et se présentant ainsi, fait monter en lui les gestes qu'il capte, saisis dans son vol(e).

\section{2. Geste et passage à l'acte : le désir}

20 Ce pourquoi le cinéaste documentaire a ceci de singulier, lui, l'intervenant, qu'il peut à la fois faire des gestes et se les voler. Par exemple Henri-François Imbert dans Sur la plage de Belfast ${ }^{29}$ filme un raccord entre une trace et son traceur qu'il est en train de faire. 
Il va en effet partir en quête de personnes filmées sur une pellicule qu'il a achetée à un brocanteur. Possédant la trace il cherche à remonter vers ceux qui ont souvenir de l'ancien présent où s'est réalisé le film, ancien présent du traçage de la trace. Nous, spectateurs, assistons au geste en train de se faire: enquête, réflexions en voix off, pérégrinations ${ }^{30}$. Le film est tout autant tracés et traces-raccords dans lesquels s'enveloppent les gestes de traçage et de coupure du cinéaste, que le tracé et le raccord du traçage aux traceur et filmés du film retrouvé, les intensités d'affect culminantes étant celles du moment de la retrouvaille du film par les filmés, intensités d'autant plus fortes que le filmeur est mort entre temps. Le geste de Sur la plage de Belfast s'est donc doublé de la réalisation d'un acte : rapporter le film trouvé. Or cet acte aurait-il été possible sans ce geste ? Le geste en train de se faire est bien celui de l'acte en tant qu'il s'en élève (il le virtualise), mais en quoi l'acte dépend-t-il du geste? Pour son désir. Le geste cinématographique relance constamment l'acte et donc le film, il le provoque car le geste en train de se faire veut continuer, il persévère indéfiniment dans son être. Arrêtant toute réalisation il est par là même sans arrêt. Le passage à l'acte donne des mains au désir du geste et redessine à chaque fois un nouveau contenu disponible. Ce que je nommais "geste d'intervention » se précise maintenant un peu mieux, le geste est d'intervention car il est désir de l'acte ${ }^{31}$. Dans Babel. Lettre à mes amis restés en Belgique, Boris Lehman est confronté à ce désir, à cette puissance gestuelle filmique qui l'emporte, qu'il ne peut arrêter et qui n'aura pas d'arrêt. «Babel » est le nom propre d'un geste, comme la tour du même nom (elle, dont la réalisation est à la fois arrêtée et sans arrêt...). Le geste cinématographique incite au passage à l'acte mais il ne peut luimême passer comme acte ${ }^{32}$. Alors s'il le faut c'est le cinéma lui-même qui passera à l'acte, entraîné par le désir du geste cinématographique comme le ciné-train d'Alexandre Medvedkine raccordant les ouvriers de la Russie communiste, le geste cinématographique alimentant leur désir d'être communistes, en actes.

Ces actes peuvent être de véritables individuations ${ }^{33}$ collectives. Les films des groupes ouvriers Medvedkine ${ }^{34}$ (Besançon, Sochaux) ont été de véritables tremplins pour individuer les travailleurs, non pas seulement parce qu'ils ont pris la caméra mais parce que les films eux-mêmes ont eu les potentiels suffisant pour les transformer. Ainsi dans À bientôt j'espère (1967-1968) le premier film du groupe qui est à vrai dire celui de professionnels «Chris Marker et Mario Marret» nous voyons une jeune femme (Suzanne) qui vient de rentrer à l'usine. Assez effacée derrière son mari nous la retrouvons métamorphosée dans un film documentaire un peu plus tardif Classe de lutte (1968) où elle est maintenant une militante très active, elle a découvert l'art (Picasso, Prévert), elle s'est émancipée en tant que femme. Le geste documentaire n'a-t-il pas contribué à la faire passer à l'acte ? Les gestes filmiques du groupe n'ont-ils pas soulevé dans leur suspens des gestes politiques émancipateurs enveloppant des singularités, prêts pour des retombées individuelles et collectives?

\section{La fiction documentaire individuante, le Tiers}

Car le cinéma documentaire a ceci de paradoxal : dégager une ligne du possible, une ligne de fiction. En s'engageant dans un réel, le cinéma documentaire dégage une ligne gestuelle qui déborde de ce réel. Car en premier lieu il y a une autonomie de cette ligne exactement comme la réalité intermédiaire des images (Simondon). Dès que le filmé est couplé au filmeur une part de ce qui est dit, fait, restera comme en suspens, passage qui 
ne passe pas, levée du geste, part immortalisée sur et par ce Tiers qu'est la pellicule et l'écran. La pellicule-écran (réception-émission) est le Tiers qui, parce qu'il n'appartient à personne, joint filmeur, filmé et spectateur. C'est le Tiers de l'« avec ». Ce pourquoi est insupportable que ce Tiers serve à conserver les traces d'enjeux privés ou à l'appropriation par et pour quelqu'un, le Tiers de l'«avec » devient alors le Tiers de l'«à moi ». Au Tiers il faut réserver la réception-émission des singularités impersonnelles enveloppées dans le geste du filmeur. Une singularité peut mettre en émoi une autre singularité que nous impliquons, elle peut faire proliférer son "autour ». Le Tiers est donc plus fondamentalement le site actif des singularités qui nous machinent dont se réclame la réalité intermédiaire des images.

Ne nous étonnons pas alors en second lieu que la ligne gestuelle, puisqu'elle se manifeste à nous au niveau de notre corps imaginacteur et de notre mémoire, puisse inciter à donner libre cours à l'imagination fabulatrice ${ }^{35}$ et déborder d'un réel. C'est cette incitation que Jean Rouch a exploité avec génie. Avec Jaguar ${ }^{36}$, son geste cinématographique va dégager d'un réel une ligne de fiction qui par le geste de coupure du montage (image/bande son) viendra en retour l'investir au point de rendre indiscernables réel et fiction, comme pour des enfants qui jouent. Jean Rouch a procédé pour cela à un décalage temporel entre l'enregistrement des images (1957) et l'enregistrement des paroles (1967) par les trois protagonistes du film qui vont fabuler, extraire à partir des images leur propre ligne de fiction. Ce sont des actes de paroles. En 1974 par Cocorico! Monsieur Poulet le filmeur "Jean Rouch » passera à l'acte. Il nous embarquera comme il est embarqué sur la ligne réelle-fictive, créatrice d'actes et funambulesque, de son geste ${ }^{37}$. Le geste documentaire traverse filmeur, filmé et spectateurs. Cette traversée peut être qualifiée de transindividuelle au sens de Gilbert Simondon. Selon lui la relation transindividuelle se déploie et s'accomplit lors d'une individuation psychique et collective. L'individuation psychique et collective implique une circulation émotionnelle (qui ici passe aussi par nous spectateurs) et une organisation active (significative), ici entre filmeur et filmés (mais nous aussi, indirectement, en tant que nous habitons les gestes du film).

Exemplaire est à ce titre le documentaire Pour la suite du monde de Pierre Perrault. Il va filmer le geste qu'il réactive : celui de l'ancienne chasse au marsouin. Si bien que son geste cinématographique peut être vu à la fois comme celui qui par son intervention induit une véritable individuation psychique et collective des habitants de l'Ile-auxCoudres (passage à l'acte) mais aussi comme celui du film de cette individuation (qui passe ainsi par nous). Des actes (physique et de paroles) se relaient et des émotions circulent.

Il fallait trouver une action... et je ne voulais pas non plus une action pour le magnétophone ou la caméra... mais une action vivante, vivable, possible, voulue, désirée par les acteurs, en dépit de la caméra. Déclencher une telle action, faire en sorte que les événements se produisent devant moi - et non pour moi - mais entre eux ou entre nous, et j'obtenais non plus un récit mais l'acte... pas un témoignage mais la vie elle-même. C'est alors que j'ai songé au marsouin. Parce que je savais la place qu'il occupe dans les désirs des gens de l'ile-aux-Coudres. Je n'avais dès lors qu'à suivre les événements, à obéir à la réalité, et à regarder vivre l'homme à la poursuite de son exploit ${ }^{38}$.

Le geste-événement documentaire de Perrault déclenche, enveloppe et prolonge une individuation psychique et collective "l'homme à la poursuite de son exploit». Et cela passe bien par la présence d'une dimension tierce, un Tiers événementiel, «les 
événements se produisent [...] entre eux ou entre nous ». Ce Tiers, dont la place est aussi occupée par la caméra, amplifie la singularité qu'est la chasse au marsouin en même temps qu'elle résonne chez chacun des protagonistes. La présence technique cinématographique est l'opérateur im-personnel (il n'est là pour personne) de l'organisation active du collectif (les filmés se prennent au jeu et font le jeu) de même que ce collectif par son individuation propre réagit sur le filmeur. Perrault est au carrefour de l'individuation technique filmique qu'il module et de l'individuation psychique et collective dans laquelle il baigne. Les deux individuations puisent dans la même source transindividuelle, comme l'appareillage du montage dont les gestes de coupure font retentir les plans dans nos mémoires vibrantes et celles qui vibreront encore, pour la suite du monde...

Gilbert Simondon, auteur de Du mode d'existence des objets techniques, n'a-t-il pas insisté sur le fait, que la relation à l'objet technique ne peut devenir adéquate que :

dans la mesure où elle arrivera à faire exister cette réalité interindividuelle collective, que nous nommons transindividuelle, parce qu'elle crée un couplage entre les capacités inventives et organisatrices de plusieurs sujets. Il y a relation de causalité et de conditionnement réciproque entre l'existence d'objets techniques nets, non aliénés, utilisés selon un statut qui n'aliène pas, et la constitution d'une telle relation transindividuelle ${ }^{39} .40$

Concluons alors avec Deleuze et Guattari que l'appareillage cinématographique du Tiers connectif de l'«avec» est une véritable machine désirante car le geste dont il est l'opération est désir de l'acte et non fantasme: «le tiers, la machine désirante en personne ${ }^{41} »$.

\section{BIBLIOGRAPHIE}

Agamben Giorgio, « Notes sur le geste », in Moyens sans fins, Paris, Rivages, 1995.

Bergson Henri, Les deux sources de la morale et de la religion, Paris, PUF, 2008.

Bresson Robert, Notes sur le cinématographe, Paris, Gallimard (Folio), 1988.

Combes Muriel, Simondon. Individu et collectivité, Paris, PUF, 1999.

Comolli Jean-Louis, Voir et pouvoir, Lagrasse, Verdier, 2004.

Deleuze Gilles, Logique du sens, Paris, Minuit, 1969.

Deleuze Gilles, Guattari Félix, Capitalisme et schizophrénie, L'anti-Oedipe, t. 1, Paris, Minuit, 1972/1973.

Jousse Marcel, Anthropologie du geste, Paris, Gallimard, 1974.

Lacan Jacques, Les quatre concepts fondamentaux de la psychanalyse, Paris, Points Seuil, 1973.

Perrault Pierre, Image et son, $\mathrm{n}^{\circ}$ 183, avril 1965.

Schmitt Jean-Claude, La raison des gestes dans l'occident médiéval, Paris, Gallimard, 1991.

Simondon Gilbert, Du mode d'existence des objets technique, Paris, Aubier, 1989. 
Simondon Gilbert, Imagination et invention, Chatou, Éd. de la transparence, 2008.

\section{NOTES}

1. Ce pourquoi une ombre chinoise animée n'est pas cinématographique puisqu'elle se réfère à un point de vue extérieur à elle, celui du spectateur.

2. Par exemple si le point de vue est celui d'un filmeur placé dans une voiture, nous projetons notre corps en l'ancrant dans le référentiel terrestre si bien que nous imaginons bien que la voiture se déplace par rapport au paysage qui l'environne et non l'inverse, ce qui est pourtant le cas depuis le filmeur. Le référentiel terrestre exprime plus généralement l'idée d'un référentiel absolu, un repos absolu, ce pourquoi il peut aussi être sup-posé dans des scènes tournées dans l'espace interstellaire. Cela ne signifie pas que ce géocentrisme du cinéma est incontournable car peut être envisagé un cinéma plus galiléen où c'est le seul point de vue du filmeur qui ordonne la multiplicité (tout mouvement filmé lui devient relatif).

3. Si bien que le filmeur est une équipe.

4. Vont ainsi apparaitre toutes les formes géométriques, les horizontales, les verticales, les lignes, qui partagent l'espace et créent sa tension propre.

5. Ce n'est pas le cas par exemple avec un dessin. Le dessin tracé suggère son traçage mais ne le présente pas avec lui.

6. Nous suivons ici Deleuze et Guattari qui qualifient de diagrammatique l'indiscernabilité entre contenu et expression dans " Mille plateaux ».

7. Nous n'envisageons plus ici que le geste de coupure du raccord et non celui des compositions par superposition ou partage.

8. Ainsi quand le montage suit et forge l'établissement d'un lieu (par ses changements de points de vue discontinus), l'unité du lieu crée un fond d'attentes comblées.

9. Évidemment dans le cas où le film est un unique plan-séquence il n'y a plus de geste de coupure.

10. Nous avons défini plus haut les opérations, elles sont les opérations techniques de l'appareillage cinématographique qui réalisent le geste, elles sont des actes, mais tout acte n'est pas une opération technique, l'acte peut être celui d'un filmé par exemple.

11. Même en direct, il y a un infime temps entre la réalisation et son enregistrement. Les ondes n'ont pas une vitesse infinie.

12. «Qu'est-ce qu'un geste? un geste de menace, par exemple? Ce n'est pas un coup qui s'interrompt. C'est bel et bien quelque chose qui est fait pour s'arrêter et se suspendre. [...] en tant que geste de menace, il s'inscrit en arrière. Cette temporalité très particulière, que j'ai définie par le terme d'arrêt, et qui crée derrière elle sa signification, c'est elle qui fait la distinction du geste et de l'acte" Jacques Lacan, Les quatre concepts fondamentaux de la psychanalyse, Paris, Points Seuil, 1973, p. 132.

13. On peut rapporter au geste pur ce que Gilles Deleuze dit de l'opération pure (distincte de son incorporation-réalisation), il est continuellement dans un présent sans épaisseur qui est bien « le présent de l'acteur, du danseur ou du mime, pur “moment” pervers. C'est le présent de l'opération pure et non de l'incorporation ", Logique du sens, Paris, Minuit, 1969, p. 197.

14. "Virtualiser» ne signifie donc surtout pas le passage à un manque de réalité. Le virtuel est réel. Cf. Gilles Deleuze « Différence et répétition », «Le pli », « Cinéma 2 » ou encore « L'actuel et le virtuel » dans Dialogues.

15. J'emprunte ce terme à Joachim Dupuis.

16. Niveaux de la réalité qui s'incluent: l'imagination est impliquée dans la perception (ce que par exemple Kant a montré avec la synthèse d'appréhension et de reproduction même s'il maintient une doctrine des facultés) comme les ondes sont impliquées par les objets massiques. 
17. Imagination et invention, Chatou, Éd. de la transparence, 2008, p.7-18. Gilbert Simondon souligne bien aussi le rôle intra-perceptif de l'image.

18. Ceci rappelle la formule de Schelling «la nature est l'esprit visible et l'esprit la nature invisible » qui noue esprit, nature et image (le visible)...

19. «Le cinéma ramène les images dans la patrie du geste. Traum und Nacht, de Beckett, en propose implicitement une belle définition: il est le rêve d'un geste. Introduire en ce rêve l'élément du réveil, telle est la tâche du cinéaste » Giorgio Agamben « Notes sur le geste ", dans Moyens sans fins, Paris, Rivages, 1995, p. 67. Marcel Jousse (1886-1961), répète plusieurs fois dans son Anthropologie du geste (Paris, Gallimard, 1974) que le cinéma deviendra de plus en plus essentiel à l'étude du geste.

20. «Il ne s'agit pas de jouer "simple", ou de jouer "intérieur", mais de ne pas jouer du tout ». Robert Bresson, Notes sur le cinématographe, Paris, Gallimard (Folio), 1988, p. 99.

21. Ibid., p. 27. C'est Robert Bresson qui souligne.

22. Par exemple dans L'argent, raconté aux enfants et à leurs parents.

23. Jean-Louis Comolli, Voir et pouvoir, Lagrasse, Verdier, 2004, p. 532.

24. Sylvain George, Qu'ils reposent en révolte, vidéo, 2005-2009.

25. Denis Gheerbrant, Marseille dans ses replis, film, 2009.

26. Jean-Louis Comolli, Voir et pouvoir p. 516.

27. Boris Lehman, Babel. Lettre à mes amis restés en Belgique, film, 1991.

28. Jean-Claude Schmitt, La raison des gestes dans l'occident médiéval, Paris, Gallimard, 1991, p. 133.

29. Éditions Montparnasse, collection "Le geste cinématographique " dirigée par Patrick Leboutte, dvd comprenant les films de H.-F. Imbert de 1996 à 2003.

30. On retrouve ce genre de démarche enquêtrice chez Claudio Piazienza, Tableau avec chutes, 1996.

31. «filmer, c'est bien, quoiqu'il en soit et d'une manière ou d'une autre, faire, défaire ou refaire le monde. Parce que je ne peux pas vivre si je ne pense pas que mon désir peut changer un rien, juste un rien, qui est tout " (Denis Gheerbrant).

32. L'acte nécessite la réalisation massique. De même que Boris Lehman se confronte à la puissance du geste cinématographique et à son impuissance à être lui-même un acte et qu'il filme cette impossibilité, pensant la conjurer par le film des opérations qui lui donnent naissance (la recherche d'un producteur, les accords et désaccords des filmés etc.), or les opérations dans le film deviennent des gestes propres au film et non plus des actes... on ne s'étonnera pas qu'un autre de ses films pose le problème de la réalisation filmique, de sa masse qui, tout en permettant le film, ne "pèse " plus dans sa substance finale et qu'il veuille aussi conjurer cette impossibilité en la filmant... ou en étant présent lui-même lors de la projection de ses films avec son appareillage massique. Homme portant son film le plus lourd, 1994. Le cinéma de Boris Lehman est travaillé par l'impossibilité du cinéma, par le désir de cette impossibilité.

33. Par individuation nous entendons la transformation individuelle. Or une individuation peut avoir eu pour condition la constitution d'un collectif. On parlera alors d'individuation collective, tout autant individuation de chaque individu que du collectif lui-même (qui se constitue par là comme entité singulière).

34. Édités aux Éditions Montparnasse, collection « Le geste cinématographique ».

35. Bergson tient à bien différencier «imagination» et «fabulation"si on entend par imagination le simple fait de se représenter quelque chose qui n'est ni un objet présent, ni une chose passée alors que la fabulation implique un récit, elle est la faculté «de créer des personnages dont nous nous racontons à nous-mêmes l'histoire ", elle implique une véritable croyance, «elle prend une singulière intensité de vie chez les romanciers et les dramaturges. Il en est parmi eux qui sont véritablement obsédés par leur héros; ils sont menés par lui plutôt qu'ils ne le mènent [...] À vrai dire, on la trouve à quelque degré chez tout le monde. Elle est très vivante chez les enfants ». Les deux sources de la morale et de la religion, Paris, PUF, 2008, p. 205-206. 
La fabulation ayant selon Bergson un sens supplémentaire puisqu'elle a une fonction sociale. «La fonction fabulatrice, innée à l'individu, a pour premier objet de consolider la société ; mais nous savons qu'elle est également destinée à soutenir l'individu lui-même, et que d'ailleurs l'intérêt de la société est là » ibid., p. 209.

36. Éditions Montparnasse, collection «Le geste cinématographique », films de 1956 à 1972.

37. Au sujet de Cocorico! Monsieur Poulet Jean Rouch raconte «Ce film a peut-être été le plus drôle à faire. Lam avant proposé un documentaire sur le commerce du poulet, nous décidons d'en faire un film de fiction réalisé par Dalarou, nouveau réalisateur multinational et tricéphale : Damouré Zika, Lam Ibrahima Dia, Jean Rouch. Nous avons été dépassés dans l'improvisation par les incidents : la voiture de Lam n'avait ni freins, ni phares, ni papiers. Ses pannes continuelles modifiaient sans cesse le scénario prévu [...] Alors l'invention était continuelle et nous n'avions aucune autre raison de nous arrêter que le manque de pellicule ou le fou rire qui faisait trembler dangereusement micros et caméras " Cités dans le dvd édités par les Éditions Montparnasse, collection « Le geste cinématographique », 2007.

38. Pierre Perrault, Image et son, $\mathrm{n}^{\circ} 183$, avril 1965.

39. Gilbert Simondon, Du mode d'existence des objets technique, Paris, Aubier, 1989, p. 245.

40. Muriel Combes, Simondon. Individu et collectivité, Paris, PUF, 1999, p. 126.

41. Gilles Deleuze, Félix Guattari, Capitalisme et schizophrénie, L'anti-Oedipe, t. 1, Paris, Minuit, 1972/1973, p. 65.

\section{RÉSUMÉS}

Cet article propose de définir le geste cinématographique par la composition de deux gestes directeurs : celui de traçage et celui de coupure qui, comme tout geste, ne supposent pas la prise de conscience pour exister. Ceci se fera sur fond, ici seulement esquissé, d'une orientation philosophique naturaliste corrélative d'une certaine philosophie du geste. La nature ondulatoire du geste cinématographique se révèlera ainsi comme présentant la nature spirituelle, affective de son geste et de tout geste, évitant de le réduire à l'acte par lequel il prend part à une effectuationréalisation. Ce geste sera mis à l'épreuve dans le champ du cinéma documentaire (qui n'est pas celui du reportage) où spécifiquement geste et passage à l'acte d'un geste se suscitent, engendrant désirs ou individuations politiques. Appareillage cinématographique et relation transindividuelle (au sens de Simondon) seront par leur réciprocité au cœur de ces engendrements, logés sur la ligne tierce des images, des gestes et des singularités, suivant laquelle peuvent être rendus indiscernables réel et fiction.

\section{INDEX}

Mots-clés : acte, coupure, documentaire, fabulation, geste, imagination, individuation, onde, réalisation, singularité, traçage, virtualisation

Personnes citées : Simondon (Gilbert), Deleuze (Gilles) 
AUTEUR

PHILIPPE ROY

Doctorant en philosophie, laboratoire d'Étude et de Recherches sur les Logiques contemporaines de la Philosophie, université de Paris 8. Membre de l'association de cinéma «Ptchila kino », Besançon. 